\title{
Paralisia obstétrica: De quem é a culpa? Uma revisão sistemática de literatura*
}

\section{Obstetric Paralysis: Who is to Blame? A Systematic Literature Review}

\author{
José Antonio Galbiatti ${ }^{1}$ Fabrício Luz Cardoso ${ }^{2}$ Marília Gabriela Palacio Galbiatti ${ }^{3}$
}

${ }^{1}$ Serviço de Ortopedia e Traumatologia, Santa Casa de Misericórdia de Marília, Faculdade de Medicina de Marília, Marília, SP, Brasil

2 Departamento de Ortopedia e Traumatologia, Faculdade de

Endereço para correspondência Fabrício Luz Cardoso, Av. Monte Carmelo, 800 - Fragata, Marília, SP, Brasil. CEP: 17519-030 Medicina de Marília, Marília, SP, Brasil

${ }^{3}$ Faculdade de Medicina, Universidade de Marília,, Marília, SP, Brasil (e-mail: fabricioramalhense@gmail.com).

Rev Bras Ortop 2020;55(2):139-146.

\section{Resumo}

Palavras-chave

- paralisia obstétrica

- ombro

- distocia

- traumatismos do nascimento
A paralisia obstétrica é classicamente definida como a lesão do plexo braquial decorrente da distócia de ombros ou das manobras executadas no parto difícil. Nas 2 últimas décadas, vários estudos comprovaram que metade dos casos de paralisia obstétrica não estão associados à distócia de ombros e levantaram outras possíveis etiologias para a paralisia obstétrica. O objetivo do presente trabalho é colher dados da literatura de revisão, artigos clássicos, sentinelas e da medicina baseada em evidências para compreender melhor os eventos envolvidos na ocorrência de paralisia obstétrica. Foi realizada uma revisão da literatura no motor de busca da PubMed (MeSH - Medical Subject Headings) com as seguintes palavras-chave: shoulder dystocia and obstetric palsy, completamente aberto, sem limites de língua ou data. Posteriormente, definimos como critério de inclusão artigos de revisão. Encontramos 21 artigos de revisão com associação dos temas descritos até 8 de março de 2018. Frente às melhores evidências existentes até o momento, está bem demonstrado que a paralisia obstétrica ocorre em partos não complicados e em partos cesáreos, e são múltiplos os fatores que podem causá-la, relativizando a responsabilidade de médicos obstetras, enfermeiras e parteiras. Procuramos, com o presente estudo, quebrar os paradigmas de que paralisia obstétrica se associa obrigatoriamente à distócia de ombros e que a sua ocorrência necessariamente implica em negligência, imperícia ou imprudência da equipe envolvida.

Trabalho desenvolvido na Faculdade de Medicina de Marília, Marília, SP, Brasil.

recebido

12 de Março de 2018

aceito

06 de Agosto de 2018
DOI https://doi.org/

10.1055/s-0039-1698800. ISSN 0102-3616.
Copyright $\odot 2020$ by Sociedade Brasileira License terms de Ortopedia e Traumatologia. Published by Thieme Revinter Publicações Ltda, Rio de Janeiro, Brazil 


\section{Keywords \\ - paralysis, obstetric \\ - shoulder \\ - dystocia \\ - birth injuries}

sentries, and evidence-based medicine to better understand the events involved in the occurrence of obstetric palsy. A literature review was conducted in the search engine PubMed (MeSH - Medical Subject Headings) with the following keywords: shoulder dystocia and obstetric palsy, completely open, boundless regarding language or date. Later, the inclusion criterion was defined as revisions. A total of 21 review articles associated with the themes described were found until March 8, 2018. Faced with the best available evidence to date, it is well-demonstrated that obstetric palsy occurs in uncomplicated deliveries and in cesarean deliveries, and there are multiple factors that can cause it, relativizing the responsibility of obstetricians, nurses, and midwives. The present study aims to break the paradigms that associate obstetric palsy compulsorily with shoulder dystocia, and that its occurrence necessarily implies negligence, malpractice or recklessness of the team involved.

\section{Introdução}

A paralisia obstétrica (PO) é definida como uma paralisia flácida parcial ou total que acomete o membro superior do recém-nascido decorrente da lesão do plexo braquial ocorrida no parto normal, e, mais mais raramente, no parto cesáreo, frequentemente associada à distócia de ombros (DO). Também é definida como paralisia devido à lesão recebida no parto, segundo os Descritores em Ciências da Saúde (DeCS). ${ }^{1}$

A DO ou distócia de bisacromial se dá quando a apresentação é cefálica e o polo cefálico já se desprendeu, porém os ombros não se soltam e não se observam quaisquer outras dificuldades $^{2}$ (- Figura $\mathbf{1}$ ).

Apesar de rara, com incidência em $<1 \%$ dos partos, a PO é uma complicação grave, cuja ocorrência aumenta consideravelmente para valores próximos de $6 \%$ em casos de fetos com peso $>4.000$ g. ${ }^{3,4}$ São descritos como fatores associados a essa intercorrência a obesidade materna, o pós-datismo e o diabetes gestacional. ${ }^{3-5}$ É importante ressaltar que mesmo em fetos de peso $<4.000 \mathrm{~g}$, a DO pode ocorrer, principalmente quando existe algum grau de desproporção entre o feto e a pelve materna. ${ }^{2}$

A DO associa-se a complicações maternas, sendo as mais comumente descritas lacerações do canal de parto, atonia uterina com hemorragia, disjunção da sínfise púbica e, eventualmente, rotura uterina. ${ }^{2}$

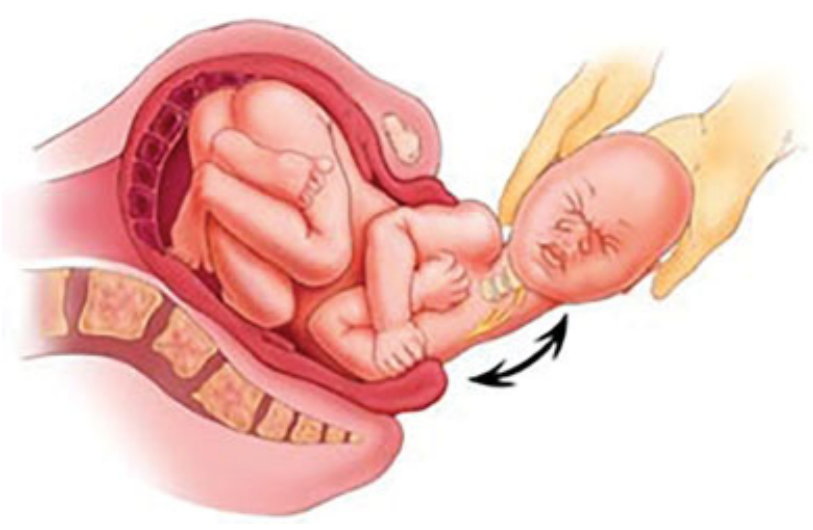

Fig. 1 Distócia de ombros.
As complicações fetais são lesões de plexo braquial e fratura de clavícula e úmero, podendo evoluir para óbito intraparto ou neonatal. 0 plexo braquial é formado pela união dos ramos anteriores das raízes de C5, C6, C7, C8 e $\mathrm{T} 1$, emergindo entre os músculos escaleno anterior e médio. Em muitos casos, recebe a contribuição de $\mathrm{C} 4$, quando é chamado de pré-fixado. A situação na qual essa contribuição é de T2 chama-se de pós-fixada. ${ }^{6}$ Em geral, o plexo braquial é lesado pela tração excessiva durante o trabalho de parto, o que pode ocorrer em até $15 \%$ dos recém-nascidos que apresentem distócia de bisacromial. ${ }^{7}$ Em grande parte dos casos, a lesão se resolve em entre 6 e 12 meses, mas casos severos podem precisar de cirurgia, com risco de dano

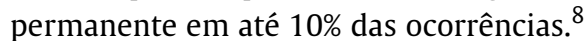

Galbiatti, ${ }^{9}$ Galbiatti et al ${ }^{6}$ e Albertoni et al, ${ }^{10}$ em estudos sobre PO, trazem bases anatômicas e fazem um breve histórico dessa lesão. Os autores destacam que esta é reconhecida desde a antiguidade na literatura médica. Foi citada brevemente por Smellie em 1764. Em 1872, Duchenne de Boulogne, em seu "Traitéde l'Électrisation Localisée", empregou o termo paralisia de origem obstétrica e descreveu a paralisia das raízes superiores. Erb, em 1874, detalhou o quadro clínico da PO do tronco superior (C5 e C6). Em 1885, Klumpke descreveu a paralisia mais rara das raízes inferiores (C8 e T1), a qual pode estar associada à síndrome de Claude Bernard-Horner.

A incidência de PO varia entre 0,1 e $0,4 \%$ de todos os nascidos vivos. Apesar dos avanços no cuidado obstétrico, a incidência de PO pode estar aumentando devido ao maior número de recém-nascidos de alto peso. Vários fatores de risco foram identificados, entre eles trabalho de parto prolongado, recém-nascido de alto peso, parto laborioso, uso de fórceps e partos prévios que cursaram com PO. A DO é o fator mecânico que resulta na lesão do tronco superior. ${ }^{11}$ Fatores de risco significantes são: peso do recém-nascido $>4.000 \mathrm{~g}$, DO e apresentações pélvicas. ${ }^{12}$

O tipo mais comum de PO, e que apresenta melhor prognóstico, é a paralisia denominada alta ou de ErbDuchenne (90\% dos casos), em que as raízes de C5-C6 são acometidas e os músculos mais frequentemente envolvidos são o supra- e infraespinhal ( - Figura 2). Há ainda a paralisia total (mista), que apresenta paralisia tanto motora quanto sensitiva de toda a extremidade do membro afetado devido a 


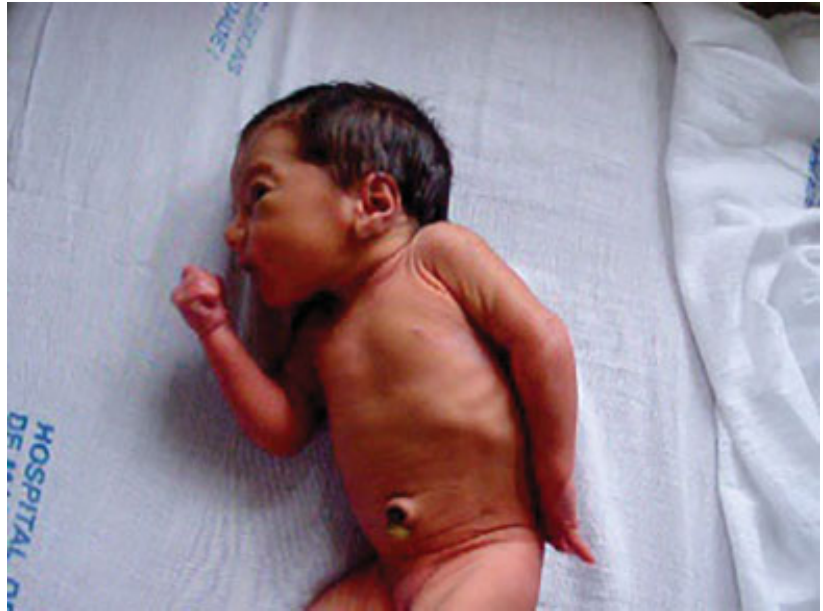

Fig. 2 Paralisia obstétrica de Erb.

lesão de todas as raízes do plexo; e a paralisia baixa ou de Klumpke (C8-T1), na qual os músculos do antebraço e mão são os mais acometidos. A gravidade da lesão depende das raízes afetadas e de sua extensão.

Galbiatti et al $^{6}$ apresentam a anatomia clássica do plexo braquial e explicam que as lesões neurais podem ser classificadas em três graus funcionais, segundo Seddon ${ }^{13}$ :

Neuropraxia: sem alteração morfológica neural, definida como bloqueio localizado de condução por alteração metabólica e representada clinicamente por paralisia motora, discreta alteração sensitiva e simpática, apresentando recuperação total ao longo de algumas semanas.

Axonotmese: interrupção axonal, sem lesão do endoneuro, levando a degeneração waleriana distal à lesão. Ocorre regeneração axonal variável orientada pela preservação do endoneuro; o tempo de recuperação depende, acima de tudo, da distância da lesão aos efetores (basicamente à placa motora muscular).

Neurotmese: determina uma lesão total do nervo com destruição das estruturas internas e externas. Não ocorre regeneração espontânea, sendo necessário o tratamento cirúrgico.

Na prática clínica, é frequente a dificuldade de classificação das lesões, principalmente da axonotmese. Deve-se considerar, ainda, em relação às lesões do plexo braquial, as avulsões ao nível da medula espinhal, também chamadas de lesões pré-ganglionares, por se encontrarem proximais aos gânglios dos neurônios motores, que não permitem reparação. Nessa situação, também ocorre lesão do ramo posterior da raiz nervosa e leva à denervação da musculatura paravertebral da região cervical.

Os autores ressaltam a importância dos diagnósticos diferenciais mais frequentes, que são a paralisia cerebral, as fraturas da clavícula e da diáfise do úmero, presentes em entre 10 e $15 \%$ dos casos em apresentação cefálica. ${ }^{6} 0$ descolamento epifisário proximal do úmero, a osteomielite neonatal, a artrite piógena do ombro e a sífilis congênita também são diagnósticos diferenciais.

A PO é uma das complicações associadas ao parto que necessita ser mais bem estudada e compreendida, pois mantém sua incidência internacional até os dias atuais, ${ }^{14}$ ocorrendo em todo o mundo, não distinguindo países ricos ou pobres, cirurgiões obstetras jovens ou com vasta experiência, de enfermeiras obstétricas ou parteiras.

Nessa conjuntura, vêm aumentado as demandas médicolegais relacionadas a eventos desfavoráveis relacionados ao parto, entre eles a PO. Neste escopo, principalmente os obstetras são responsabilizados ou culpados por esses eventos ocorridos durante sua atividade profissional. Configura-se em um clássico paradigma que, na última década, com a evolução da medicina baseada em evidência (MBE), está sendo quebrado. Há atualmente novos estudos comprovando novas etiologias como origem congênita, intrauterina, e causadas pelas forças endógenas do parto, dentre outras. Já em 1997, Paradiso et $\mathrm{al}^{15}$ publicam importante artigo que trata do exame de eletroneuromiografia (ENMG) ao nascimento, demonstrando que a lesão do plexo braquial poderia ter ocorrido intraútero. Os autores apresentam relato sobre uma criança de 18 dias com lesão de C5-C6 cujo exame de ENMG era compatível com uma lesão antiga, datada de antes do nascimento. Há casos em que certas crianças nascem com paralisia sem anormalidade no parto, sem distócia e sem nenhuma manobra particular.

A definição clássica traz sinais de que a causa seria dos partos distócicos ou das manobras do parto executadas pelo médico assistente. $\mathrm{O}$ presente trabalho tem por objetivo colher dados da literatura de revisão, artigos clássicos, sentinelas e da MBE para que os profissionais envolvidos com essa entidade, dentre eles o perito médico, tenham subsídios científicos suficientes para compreender melhor os eventos ocorridos durante o parto e identificar realmente de quem é a responsabilidade da PO.

Vamos, com esta revisão, à luz dos mais recentes estudos, mudar a direção, pelo menos parcialmente, das causas da PO.

\section{Métodos}

Foi realizada uma pesquisa bibliográfica no motor de busca da PubMed (Medical Subject Headings [MeSH, na sigla em inglês]) com as seguintes palavras-chave: shoulder dystocia and obstetric palsy, completamente aberto, sem limites de língua ou data. Encontramos 87 artigos. Posteriormente, definimos como critério de inclusão artigos de revisão, dos quais restaram 22 artigos de revisão com associação aos temas descritos até 8 de março de 2018. Excluímos 1 artigo, por se tratar de um mesmo estudo publicado em periódico diferente sob a forma de versão compacta, restando, portanto, 21 artigos.

Foram atribuídos a esses 21 artigos números de ordem conforme a cronologia crescente da publicação. Os artigos foram caracterizados quanto aos principais pontos relevantes, com os seguintes descritores: distocia de ombros, parto cesáreo, prevenção, histórico, etiopatogenia e médico-legal. Quando incluímos no motor de busca descritores relacionados especificamente a perícia médica, por exemplo, os descritores expert ou expert testimony, além de número limitado de artigos, estes entravam em temas não incluídos em nossa proposta científica. 
Realizamos uma revisão manual da bibliografia dos 21 artigos que originaram nossa revisão e adicionamos à nossa bibliografia os artigos que foram citados na maioria das publicações e também os artigos considerados clássicos ou sentinela (nestes não foram considerados o ano de publicação ou a língua). Com esse conceito, foram valorizados os artigos com cunho médico-legal.

\section{Resultados}

Foram incluídos 87 artigos com base nos critérios de inclusão referidos anteriormente, excluindo-se 66 que não tinham características de revisão ou eram publicações repetidas dos mesmos autores em outro periódico em versão compacta. Efetivamente, foram utilizados como base 21 artigos, 17 de língua inglesa, 2 de língua francesa e 2 de língua alemã.

Os resultados encontrados mostram que não há artigos com níveis de evidência adequados, sendo sua maioria de revisão da literatura.

No tocante ao ano da publicação, houve distribuição variável de 2000 a 2016 (-Tabela 1). ${ }^{14,16-34}$

Todos os estudos principais foram de revisão de literatura, pois esta é uma das premissas do método, e o número de artigos da bibliografia dos mesmos variou entre 11 a 121 artigos revisados.
Na - Tabela 2, encontram-se os dados referentes ao número de ordem, tipo de estudo, número de bibliografias utilizadas por cada artigo, pontos mais relevantes e conclusões do estudo.

\section{Discussão}

A PO é classicamente definida como paralisia flácida parcial ou total que acomete o membro superior decorrente da lesão do plexo braquial ocorrida no parto. Esse conceito está presente tanto nos livros-texto ortopédicos quanto obstétricos ${ }^{2,6}$, porém a literatura das últimas 2 décadas tem mostrado que mais da metade dos casos não estão associados à DO. ${ }^{14,23,35}$ Outras etiologias têm sido consideradas, como origem congênita, intrauterina, e as causadas pelas forças endógenas do parto, dentre outras.

Atualmente, temos várias evidências de que muitos outros fatores podem estar envolvidos na causa da $\mathrm{PO}$, sem nenhuma relação com as manobras de parto executadas pelos obstetras.

Zaki et al, $^{36}$ em estudo sobre lesão familiar congênita de paralisia do plexo braquial, apresentam um relatório de dois níveis sobre famílias egípcias afetadas ao nascimento, caracterizada como relativamente incomum e quase um transtorno esporádico. Mollica et al $^{37}$ descrevem uma família siciliana que apresenta lesão congênita do plexo braquial severa e sugerem que o gene tem herança autossômica dominante com

Tabela 1 Artigos selecionados

\begin{tabular}{|c|c|c|c|c|c|}
\hline Ordem & Autoria & Periódico & Ano & País & Língua \\
\hline 1 & Beller $^{14}$ & Z Geburtshilfe Neonatol & 2000 & Alemanha & Alemão \\
\hline 2 & Sandmire et $\mathrm{al}^{35}$ & Birth & 2002 & EUA & Inglês \\
\hline 3 & Sandmire et al $^{16}$ & Int J Gynaecol Obstet & 2002 & EUA & Inglês \\
\hline 4 & Krause et al ${ }^{17}$ & Z Geburtshilfe Neonatol & 2005 & Alemanha & Alemão \\
\hline 5 & Gherman et al $^{18}$ & Am J Obstet Gynecol & 2006 & EUA & Inglês \\
\hline 6 & Hankins et al ${ }^{19}$ & Semin Perinatol & 2006 & EUA & Inglês \\
\hline 7 & Gurewitsch $^{20}$ & Clin Obstet Gynecol & 2007 & EUA & Inglês \\
\hline 8 & Allen $^{21}$ & Clin Obstet Gynecol & 2007 & EUA & Inglês \\
\hline 9 & Jevitt et $\mathrm{al}^{22}$ & J Perinat Neonatal Nurs & 2008 & EUA & Inglês \\
\hline 10 & Doumouchtsis et al ${ }^{23}$ & Obstet Gynecol Surv & 2009 & Inglaterra & Inglês \\
\hline 11 & O'Shea et al ${ }^{24}$ & Semin Perinatol & 2010 & EUA & Inglês \\
\hline 12 & Doumouchtsis et $\mathrm{al}^{25}$ & Ann N Y Acad Sci. & 2010 & Inglaterra & Inglês \\
\hline 13 & Anderson $^{26}$ & Prim Care Clin Office Pract & 2012 & EUA & Inglês \\
\hline 14 & Abzug et $a^{27}$ & Orthop Clin North Am & 2014 & EUA & Inglês \\
\hline 15 & Stitely et $\mathrm{al}^{28}$ & Seminars in Perinatology & 2014 & Nova Zelândia/EUA & Inglês \\
\hline 16 & Chauhan et al ${ }^{29}$ & Seminars in Perinatology & 2014 & EUA & Inglês \\
\hline 17 & Ouzounian ${ }^{30}$ & Seminars in Perinatology & 2014 & EUA & Inglês \\
\hline 18 & Legendre et $\mathrm{al}^{31}$ & J Gynecol Obstet Biol Reprod & 2015 & França & Fancês \\
\hline 19 & Schmitz $^{32}$ & J Gynecol Obstet Biol Reprod & 2015 & França & Francês \\
\hline 20 & Hill et $\mathrm{al}^{33}$ & Womens Health & 2016 & EUA & Inglês \\
\hline 21 & Sentilhes et $\mathrm{al}^{34}$ & Eur J Obstet Gynecol Reprod Biol & 2016 & França & Inglês \\
\hline
\end{tabular}

Encontram-se descritos os dados referentes aos 21 artigos de revisão em ordem cronológica crescente, com autoria, nome do periódico, ano da publicação, país de origem dos autores e língua da publicação. 
Tabela 2 Dados detalhados dos artigos

\begin{tabular}{|c|c|c|c|c|}
\hline Ordem & $\begin{array}{l}\text { Tipo de } \\
\text { Estudo }\end{array}$ & $\begin{array}{l}\mathrm{N}^{\circ} \text { de } \\
\text { Bibliografias }\end{array}$ & $\begin{array}{l}\text { Pontos } \\
\text { Relevantes }\end{array}$ & Conclusões \\
\hline 1 & Revisão & 86 & Médico-legal & $\begin{array}{l}\text { O grande número de POs sem distócia não permite afirmar que a PO é } \\
\text { causada pelo obstetra. }\end{array}$ \\
\hline 2 & Revisão & 22 & Histórico & $\begin{array}{l}\text { PO ocorre em partos sem distócia }{ }^{36} \text {; a experiência do cirurgião indifere } \\
\text { na incidência de } \mathrm{PO}^{37} \text {; evidência indireta estabelece que as forças } \\
\text { propulsoras maternas como a causa mais provável da paralisia de Erb. }\end{array}$ \\
\hline 3 & Revisão & 17 & $\begin{array}{l}\text { Distócia } \\
\text { de Ombros }\end{array}$ & $\begin{array}{l}\text { A causa mais provável de paralisia com e sem DO é o esforço materno } \\
\text { devido às forças expulsivas no parto }\end{array}$ \\
\hline 4 & Revisão & 86 & Médico-legal & $\begin{array}{l}\text { A causa-efeito clara entre DO e lesões do plexo braquial não existe em } \\
\text { todos os casos, embora a DO esteja geralmente associada a pareceres } \\
\text { médico-legais obstétricos. }\end{array}$ \\
\hline 5 & Revisão & 121 & $\begin{array}{l}\text { Distócia } \\
\text { de Ombros }\end{array}$ & $\begin{array}{l}\text { Há risco significativamente aumentado de distócia de ombros com o } \\
\text { peso ao nascer e este aumenta linearmente; o pré-natal e o pré-parto não } \\
\text { apresentam fatores de risco estatisticamente significativos para a } \\
\text { previsão de DO; parto cesáreo profilático ou a indução medicamentosa } \\
\text { do trabalho de parto em pacientes não diabéticas devido a suspeita de } \\
\text { macrossomia fetal não tem mostrado alterar a incidência de DO. }\end{array}$ \\
\hline 6 & Revisão & 43 & Parto Cesáreo & $\begin{array}{l}\text { Cesariana realizada em todas as mulheres com } 39 \text { semanas de gestação } \\
\text { reduziria substancialmente a ocorrência tanto transitória como perma- } \\
\text { nente de lesão do plexo braquial, encefalopatia neonatal, morte intra- } \\
\text { parto e intrauterina. }\end{array}$ \\
\hline 7 & Revisão & 74 & Prevenção & $\begin{array}{l}\text { A manipulação fetal parece ser o melhor método para a resolução atraumática } \\
\text { do parto vaginal complicado, pois requer menos tração para concluí-lo. }\end{array}$ \\
\hline 8 & Revisão & 69 & Etiopatogenia & $\begin{array}{l}\text { Os médicos precisam ser treinados para abrandar e calibrar a tração, pois } \\
\text { a tendência natural é aumentá-la quando se deparam com parto difícil. } \\
\text { A tração axial deve ser utilizada mas deve-se limitar a flexão lateral da } \\
\text { cabeça. }\end{array}$ \\
\hline 9 & Revisão & 27 & Prevenção & $\begin{array}{l}\text { DO não é previsível; enfermeiros perinatais são úteis no pré-natal para } \\
\text { evitar ganho de peso excessivo e auxiliar no controle glicêmico e durante } \\
\text { o parto na função de cronometrista uma vez diagnosticada a DO. }\end{array}$ \\
\hline 10 & Revisão & 99 & Etiopatogenia & $\begin{array}{l}\text { A maioria das crianças com lesões do plexo braquial não apresentam } \\
\text { fatores de risco conhecidos; forças endógenas são de } 4 \text { a } 9 \text { vezes maiores } \\
\text { que aquelas aplicadas pelo obstetra durante a DO, segundo modelos } \\
\text { matemáticos. }\end{array}$ \\
\hline 11 & Revisão & 95 & $\begin{array}{l}\text { Distócia } \\
\text { de Ombros }\end{array}$ & $\begin{array}{l}\text { Gestantes com cesariana prévia, qual melhor opção de parto? Não há } \\
\text { estudos randomizados disponíveis que se relacionem diretamente com a } \\
\text { escolha do método de parto. }\end{array}$ \\
\hline 12 & Revisão & 51 & Prevenção & $\begin{array}{l}\text { DO é fator de risco para lesão do plexo braquial (aumenta em 100x risco } \\
\text { de PO), mas é imprevisível; proporção significativa de lesão de plexo é } \\
\text { secundária a ferimentos no útero. }\end{array}$ \\
\hline 13 & Revisão & 44 & $\begin{array}{l}\text { Distócia } \\
\text { de Ombros }\end{array}$ & $\begin{array}{l}\text { FR materno, fetal e do parto têm baixo valor preditivo; distócia de } \\
\text { ombros ocorre mais comumente em pacientes sem FR identificados. }\end{array}$ \\
\hline 14 & Revisão & 46 & $\begin{array}{l}\text { Distócia } \\
\text { de Ombros }\end{array}$ & $\begin{array}{l}\text { A paralisia de parto do plexo braquial pode resultar em déficits perma- } \\
\text { nentes ao longo da vida e continua a ser comum apesar dos avanços em } \\
\text { cuidados obstétricos. Os resultados a longo prazo das recomendações } \\
\text { atuais sobre o tratamento permanecem desconhecidos. }\end{array}$ \\
\hline 15 & Revisão & 28 & $\begin{array}{l}\text { Distócia } \\
\text { de Ombros }\end{array}$ & $\begin{array}{l}\text { Algumas manobras e algoritmos podem ser utilizados no gerenciamento } \\
\text { das distócias de ombro. A partir de estudos entre mulheres cujo parto é } \\
\text { complicado pela distócia do ombro, há evidências científicas consider- } \\
\text { áveis de que a manobra de todos os tempos é efetiva para a liberação dos } \\
\text { ombros fetais. }\end{array}$ \\
\hline 16 & Revisão & 80 & $\begin{array}{l}\text { Distócia } \\
\text { de Ombros }\end{array}$ & $\begin{array}{l}\text { O conhecimento epidemiológico da incidência, prevalência e alterações } \\
\text { temporais da NBPP deve auxiliar o clínico, evitar intervenções desne- } \\
\text { cessárias e ajudar a formular políticas de saúde baseadas em evidências. } \\
\text { A natureza extremamente infreqüente da NBPP permanente requer um } \\
\text { estudo multicêntrico para melhorar nossa compreensão dos fatores } \\
\text { antecedentes e reduzir a sequela de longo prazo. }\end{array}$ \\
\hline
\end{tabular}


Tabela 2 (Continued)

\begin{tabular}{|c|c|c|c|c|}
\hline Ordem & $\begin{array}{l}\text { Tipo de } \\
\text { Estudo }\end{array}$ & $\begin{array}{l}\mathrm{N}^{\circ} \text { de } \\
\text { Bibliografias }\end{array}$ & $\begin{array}{l}\text { Pontos } \\
\text { Relevantes }\end{array}$ & Conclusões \\
\hline 17 & Revisão & 26 & $\begin{array}{l}\text { Distócia } \\
\text { de Ombros }\end{array}$ & $\begin{array}{l}\text { Os fatores de risco históricos para a ocorrência de NBPP, sejam eles } \\
\text { estudados sozinhos ou em combinação, não foram mostrados como } \\
\text { preditores confiáveis. A maioria dos casos de NBPP ocorre em mulheres } \\
\text { com crianças }<4,500 \mathrm{~g} \text { que não são diabéticas e não têm outros fatores } \\
\text { de risco identificáveis. Além disso, a cesariana reduz, mas não elimina } \\
\text { completamente o risco de NBPP. }\end{array}$ \\
\hline 18 & Revisão & 23 & $\begin{array}{l}\text { Distócia } \\
\text { de Ombros }\end{array}$ & $\begin{array}{l}\text { Quanto à prevenção de complicações da distócia do ombro, o treina- } \\
\text { mento prático usando manequins está associado a melhorias na } \\
\text { administração de distócia do ombro do que ao treinamento usando o } \\
\text { tutorial em vídeo. O ensino de simulação para o tratamento da distócia } \\
\text { do ombro é encorajado para o treinamento inicial e contínuo dos vários } \\
\text { atores na sala de nascimento (acordo profissional). }\end{array}$ \\
\hline 19 & Revisão & 55 & Prevenção & $\begin{array}{l}\text { Para evitar a distócia do ombro e suas complicações, duas medidas são } \\
\text { propostas. A indução do trabalho de parto é recomendada em caso de } \\
\text { macrossomia iminente se o colo do útero for favorável e idade gesta- } \\
\text { cional > } 39 \text { semanas (Consenso profissional). A administração de } \\
\text { cesárea é recomendada antes do trabalho de parto no caso de (I) } \\
\text { feto }>4,500 \mathrm{~g} \text { se associado a diabetes materna, (II) feto }>5000 \mathrm{~g} \text { na } \\
\text { ausência de diabetes materno e, (III) durante o trabalho de parto, em } \\
\text { caso de macrossomia fetal e falha no progresso no segundo estágio, } \\
\text { quando a cabeça fetal está acima de uma posição + } 2 \text {. A cesariana deve } \\
\text { ser discutida quando o histórico da distócia do ombro tem sido associado } \\
\text { a complicações neonatais ou maternas graves (consenso profissional). }\end{array}$ \\
\hline 20 & Revisão & 94 & $\begin{array}{l}\text { Distócia } \\
\text { de Ombros }\end{array}$ & $\begin{array}{l}\text { A distócia do ombro pode ser prevenida através da realização de } \\
\text { cesariana preventiva em casos de risco muito alto, mas a nossa capaci- } \\
\text { dade de identificar esses casos ainda é limitada. O diagnóstico rápido e o } \\
\text { manejo da distócia do ombro quando ocorre é a chave para prevenir } \\
\text { seqüelas neurológicas permanentes. O gerenciamento requer os esfor- } \\
\text { ços coordenados de uma equipe com as habilidades necessárias. O líder } \\
\text { da equipe deve direcionar o gerenciamento e instituir uma série de } \\
\text { manobras para libertar o feto com risco mínimo para ele e para a mãe. } \\
\text { É necessária uma compreensão completa da anatomia pélvica e fetal } \\
\text { relevante, bem como dos mecanismos através dos quais a distócia pode } \\
\text { ser resolvida. }\end{array}$ \\
\hline 21 & Revisão & 11 & $\begin{array}{l}\text { Distócia } \\
\text { de Ombros }\end{array}$ & $\begin{array}{l}\text { Nenhum estudo provou que a correção dos fatores de risco (exceto } \\
\text { diabetes gestacional) reduziria o risco de DO. A atividade física é } \\
\text { recomendada antes e durante a gravidez para reduzir a ocorrência de } \\
\text { alguns fatores de risco. A implementação de treinamento prático com } \\
\text { simulação para todos os prestadores de cuidados na sala de parto está } \\
\text { associada a uma redução significativa na lesão neonatal, mas não } \\
\text { maternal. A DO continua a ser uma emergência obstétrica imprevisível. } \\
\text { Todos os médicos e parteiras devem conhecer e executar manobras } \\
\text { obstétricas, se necessário, rapidamente, mas com calma. }\end{array}$ \\
\hline
\end{tabular}

Abreviações: DO, distócia de ombros; FR, fatores de risco; NBPP, paralisia do plexo braquial neonatal; PO, paralisia obstétrica.

Dados referentes ao número de ordem, tipo de estudo, número de bibliografias consultadas no artigo, pontos mais relevantes e conclusão(ões) do estudo.

penetrância reduzida. Os autores relatam que herança ligada ao X com expressão em mulheres heterozigóticas não pode ser descartada.

As malformações uterinas e as forças propulsivas na segunda fase do parto constituem os principais fatores etiológicos para as lesões do plexo, ${ }^{16,18,25,35,38,39}$ principalmente nos casos sem DO.

Entre as causas uterinas, podemos destacar as malformações uterinas tais como miomas, septo intrauterino ou útero bicorno. ${ }^{25}$ A mal adaptação intrauterina (por exemplo, oligodrâmnio) pode estar relacionada à diminuição da resistência dos feixes nervosos do plexo braquial ou estruturas da cintura escapular, levando à lesão do plexo. ${ }^{40}$
Em relação ao parto vaginal, podemos dividi-lo didaticamente em três fases: a primeira delas é a dilatação e corresponde ao abaixamento do feto no eixo de encaixe na pelve óssea. A segunda fase é a de expulsão, que dura entre 30 minutos e 2 horas.

A segunda fase se subdivide em duas etapas: a primeira é a conclusão da descida e rotação da apresentação, e a segunda é a descida propriamente dita, na qual a pressão abdominal deve ser controlada e dirigida com as contrações uterinas para que haja a expulsão do feto. ${ }^{2} \mathrm{Na}$ segunda etapa é que pode ocorrer a DO e as lesões do plexo devido à compressão do ombro posterior contra o promontório sacral durante as contrações uterinas. Segundo modelos matemáticos, as 
forças expulsivas são quatro a nove vezes maiores que a tração exercida pelo obstetra. ${ }^{25,35}$

A terceira e última fase do parto começa ao nascimento e termina quando a placenta dequita.

Devido a esses novos conhecimentos, Gherman et al, ${ }^{39}$ já em 1998, em sua revisão, afirmavam que evidências indiretas estabeleciam as forças propulsoras maternas como a causa mais provável da paralisia de Erb, tanto nos casos com DO associada quanto nos casos sem esta, pois as técnicas de manipulação fetal direta para a resolução da DO não se associavam a maiores taxas de lesão do plexo, fraturas de úmero ou clavícula. Sandmire et al, ${ }^{16}$ em 2002, Gherman et $\mathrm{al}^{18}{ }^{18}$ em 2006, Doumouchtsis et al, ${ }^{25}$ em 2010, e Abzug et al, 27 em 2014, respectivamente, evidenciaram esses conhecimentos em suas revisões, dando informações suficientes para concluir-se que a recente ênfase dada à MBE abalou muitos dos mitos e equívocos em torno da DO. Portanto, o peso imputado ao médico assistente no parto devido a uma lesão do plexo braquial necessita ser revisto.

Os principais fatores de risco para lesão do plexo braquial são: macrossomia fetal (peso fetal $>4.000 \mathrm{~g}$ ), apresentação pélvica e DO. ${ }^{2,6,11,12,19,21,26,35}$ A obesidade materna, diabetes materno ou gestacional, aumento excessivo de peso durante a gravidez, sexo masculino do feto, prematuridade, história pregressa de macrossomia fetal, partos distócicos anteriores, multiparidade, instrumentação com fórceps, idade materna avançada e nascimento pós-termo são considerados fatores de risco secundários. ${ }^{18,21,39,40}$ Ouzounian $^{30}$ defende que a cesariana reduz, mas não elimina completamente o risco de lesão do plexo braquial.

Apesar das múltiplas análises de fatores de risco na literatura, DO não pode ser prevista ou evitada, pois os métodos precisos para identificar quais os fetos que irão experimentar essa complicação não existem. Os dados encontrados no pré-natal e pré-parto têm baixo valor preditivo. ${ }^{18,22,24,26} \mathrm{O}$ exame ultrassonográfico realizado tardiamente na gestação também apresenta baixa sensibilidade, com pouca precisão sobre a estimativa do peso ao nascimento - há uma tendência geral a superestimá-lo. Devemos salientar ainda que a DO ocorre mais comumente em pacientes sem fatores de risco. ${ }^{26}$

Como o risco de DO é diretamente proporcional ao aumento do peso do feto, ${ }^{39}$ tem-se proposto a cesariana profilática em casos selecionados. ${ }^{32,33}$ Segundo a Sociedade Americana de Ginecologia e Obstetrícia (ACOG, na sigla em inglês) e Schmitz, ${ }^{32}$ devemos considerá-la em fetos de mães diabéticas com estimativa de peso $>4.500 \mathrm{~g}$ ou fetos de mães sem fatores de risco com $>5.000 \mathrm{~g}$. Outros autores, como Hankins et $\mathrm{al}^{19}$ recomendam em fetos de mães diabéticas com estimativas de peso entre 4.000 e $4.250 \mathrm{~g}$ ou $>4.000 \mathrm{~g}$ nas gestações de mulheres não diabéticas.

Uma melhor compreensão das causas da PO se faz necessária, uma vez que as demandas médico-legais têm aumentado em todo o mundo, e, na maioria das vezes em que há perda permanente da função do membro superior, o médico assistente é obrigado a indenizar a família da criança por imperícia. Segundo a Associação de Seguros Médicos da América, de janeiro de 1985 a dezembro de 2001, o valor médio pago por indenizações relacionadas à lesão do plexo braquial foi de US\$301.000,00 (aproximadamente quatro vezes mais que o valor médio pago devido à imperícia por outras causas no período), num valor total de US\$54 milhões. Os valores indenizatórios foram maiores para médicos assistentes de instituições de ensino. ${ }^{38}$

Para evitar essas situações litigiosas, Noble ${ }^{41}$ orienta realizar registros precisos e detalhados para que haja uma defesa bem-sucedida. Porém, o fundamental continua sendo a relação médico-paciente. Hickson et $\mathrm{al}^{42}$ demonstraram em seu estudo que 70\% das mães que processaram os obstetras devido a lesões perinatais em seus recém-nascidos queixaram-se que esses profissionais não as informaram adequadamente acerca das possíveis lesões permanentes no desenvolvimento neuropsicomotor de seus filhos. Informar os riscos inerentes ao parto, como o fato de que lesões do plexo braquial não são necessariamente decorrentes de tocotraumatismo, e tentar entender os medos e anseios da paciente são essenciais para minimizar as questões médico-legais. ${ }^{36}$

A capacitação da equipe cirúrgica quanto à compreensão completa da anatomia pélvica e fetal, bem como dos mecanismos, algoritmos e manobras através dos quais a distócia pode ser resolvida, o conhecimento epidemiológico da incidência, prevalência e alterações temporais da paralisia do plexo braquial neonatal, são de fundamental importância para o bom gerenciamento de um centro obstétrico. ${ }^{19,29,32-34}$ Legendre et $\mathrm{al}^{31}{ }^{31}$ defendem em seu estudo o treinamento prático inicial e contínuo dos vários atores na sala de nascimento usando manequins, que segundo os autores está associado a melhorias na administração de distócia do ombro do que ao treinamento usando o tutorial em vídeo.

\section{Considerações finais}

Acreditamos que com a presente revisão da literatura podemos sem sombra de dúvidas auxiliar a quebrar o paradigma estabelecido desde a antiguidade de que essa lesão seria causada única e exclusivamente por imperícia médica. A literatura está mudando sua direção, demonstrando que há outras múltiplas causas ou co-causas para a ocorrência de paralisia obstétrica, em algumas delas sem nenhum envolvimento da equipe médica.

Portanto, sobre de quem é a responsabilidade da PO, no momento há evidências indiretas que estabelecem que as forças propulsoras maternas (endógenas) podem ser a causa mais provável da paralisia do plexo braquial no nascimento, nos partos sem DO e crianças com peso $<4.000$ g. A PO pode ter origem intraútero, genética, ou postural, sendo importante para a proteção médico-legal a complementação de relatórios do prontuário médico, como também a realização de exame de ENMG até 21 dias após o nascimento para definir se esta ocorreu intraútero ou no nascimento. Há ainda a necessidade de novas publicações bem embasadas e prospectivas com MBE para melhor definir qual o real ou os reais responsáveis pela ocorrência de PO.

Conflito de Interesses

Os autores declaram não haver conflito de interesses. 


\section{Referências}

1 Descritores em Ciências da Saúde (DeCS) [Internet]. ed. 2017. São Paulo (SP): BIREME / OPAS / OMS. 2017 [acesso em $2017 \mathrm{dez} 04$ ]. Disponível em: http://decs.bvsalud.org

2 Francisco RP, Fonseca ES, Sapienza DS. Parto e puerpério. In: Zugaib obstetricía. Barueri: Manole; 2008:307-529

3 Srofenyoh EK, Seffah JD. Prenatal, labor and delivery characteristics of mothers with macrosomic babies. Int J Gynaecol Obstet 2006;93(01):49-50

4 Piasek G, Starzewski J, Chil A, et al. [Analysis of labour and perinatal complications in case of foetus weight over $4000 \mathrm{~g}$ ]. Wiad Lek 2006;59(5-6):326-331

5 Seeho SK, Smith C, Mcelduff A, Morris JM. Diabetes Res Clin Pract 2007;77(02):263-268

6 Galbiatti JA, Falloppa F. Paralisia obstétrica. In: Herbert S, Xavier R, Pardini Junior AG, Barros Filho TEP, eds. Ortopedia e traumatologia: princípios e prática. 3a ed. Porto Alegre: Artmed; 2003: 830-838

7 Baxley EG, Gobbo RW. Shoulder dystocia. Am Fam Physician 2004; 69(07):1707-1714

8 Athukorala C, Middleton P, Crowther CA. Intrapartum interventions for preventing shoulder dystocia. Cochrane Database Syst Rev 2006;(04):CD005543

9 Galbiatti JA. Paralisia obstétrica. In: Faloppa F, Albertoni WM, coord. Guia de Medicina Ambulatorial e Hospitalar da UNIFESPEPM, Ortopedia e Traumatologia. São Paulo: Manole; 2008: 273-284

10 Albertoni WM, Galbiatti JA, Canedo AC, Merlotti M. Estudo anatômico do plexo braquial na criança até os seis meses de idade. Rev Bras Ortop 1994;29(03):162-169

11 Sawyer JR. Brachial plexus palsy. In: Canale ST, Beaty JH, editors. Campbell's operative orthopaedics. 12th ed. Philadelphia: Elsevier; 2013:1323-1330

12 Slooff AC. Obstetric brachial plexus lesions and their neurosurgical treatment. Clin Neurol Neurosurg 1993;95(Suppl):S73-S77

13 Seddon HJ. Three types of nerve injury. Brain Oxford 1943;(66):37

14 Beller FK. [Incidence and etiology of brachial plexus upper arm paralysis in newborn infants. A review and basic principles for legal assessment]. Z Geburtshilfe Neonatol 2000;204(05):163-169

15 Paradiso G, Grañana N, Maza E. Prenatal brachial plexus paralysis. Neurology 1997;49(01):261-262

16 Sandmire HF, DeMott RK. Erb's palsy without shoulder dystocia. Int J Gynaecol Obstet 2002;78(03):253-256

17 Krause M, Feige A. [Shoulder dystocia from a legal standpoint]. Z Geburtshilfe Neonatol 2005;209(06):201-209

18 Gherman RB, Chauhan S, Ouzounian JG, Lerner H, Gonik B, Goodwin TM. Shoulder dystocia: the unpreventable obstetric emergency with empiric management guidelines. Am J Obstet Gynecol 2006;195(03):657-672

19 Hankins GD, Clark SM, Munn MB. Cesarean section on request at 39 weeks: impact on shoulder dystocia, fetal trauma, neonatal encephalopathy, and intrauterine fetal demise. Semin Perinatol 2006;30(05):276-287

20 Gurewitsch ED. Optimizing shoulder dystocia management to prevent birth injury. Clin Obstet Gynecol 2007;50(03):592-606

21 Allen RH. On the mechanical aspects of shoulder dystocia and birth injury. Clin Obstet Gynecol 2007;50(03):607-623
22 Jevitt CM, Morse S, O'Donnell YS. Shoulder dystocia: nursing prevention and posttrauma care. J Perinat Neonatal Nurs 2008; 22(01):14-20

23 Doumouchtsis SK, Arulkumaran S. Are all brachial plexus injuries caused by shoulder dystocia? Obstet Gynecol Surv 2009;64(09): 615-623

24 O'Shea TM, Klebanoff MA, Signore C. Delivery after previous cesarean: long-term outcomes in the child. Semin Perinatol 2010;34(04):281-292

25 Doumouchtsis SK, Arulkumaran S. Is it possible to reduce obstetrical brachial plexus palsy by optimal management of shoulder dystocia? Ann N Y Acad Sci 2010;1205:135-143

26 Anderson JE. Complications of labor and delivery: shoulder dystocia. Prim Care 2012;39(01):135-144

27 Abzug JM, Kozin SH. Evaluation and management of brachial plexus birth palsy. Orthop Clin North Am 2014;45(02):225-232

28 Stitely ML, Gherman RB. Shoulder dystocia: management and documentation. Semin Perinatol 2014;38(04):194-200

29 Chauhan SP, Blackwell SB, Ananth CV. Neonatal brachial plexus palsy: incidence, prevalence, and temporal trends. Semin Perinatol 2014;38(04):210-218

30 Ouzounian JG. Risk factors for neonatal brachial plexus palsy. Semin Perinatol 2014;38(04):219-221

31 Legendre G, Bouet PE, Sentilhes L. Place de la simulation pour réduire la morbidité néonatale et maternelle secondaire à une dystocie des épaules. J Gynecol Obstet Biol Reprod (Paris) 2015;44 (10):1285-1293

32 Schmitz T. Modalités de l'accouchement dans la prévention de la dystocie des épaules en cas de facteurs de risque identifiés. J Gynecol Obstet Biol Reprod (Paris) 2015;44(10):1261-1271

33 Hill MG, Cohen WR. Shoulder dystocia: prediction and management. Womens Health (Lond) 2016;12(02):251-261

34 Sentilhes L, Sénat MV, Boulogne AI, et al. Shoulder dystocia: guidelines for clinical practice from the French College of Gynecologists and Obstetricians (CNGOF). Eur J Obstet Gynecol Reprod Biol 2016;203:156-161

35 Sandmire HF, DeMott RK. Erb's palsy causation: a historical perspective. Birth 2002;29(01):52-54

36 Zaki MSI, el Sabbagh MH, Aglan MS. Familial congenital brachial palsy: a report of two affected Egyptian families. Genet Couns 2004;15(01):27-36

37 Mollica F, Li Volti S, Grasso A, De Simone D. Familial congenital brachial palsy. Am J Med Genet 1991;41(03):322-324

38 McAbee GN, Ciervo C. Medical and legal issues related to brachial plexus injuries in neonates. J Am Osteopath Assoc 2006;106(04): 209-212

39 Gherman RB, Ouzounian JG, Goodwin TM. Obstetric maneuvers for shoulder dystocia and associated fetal morbidity. Am J Obstet Gynecol 1998;178(06):1126-1130

40 Alfonso DT. Causes of neonatal brachial plexus palsy. Bull NYU Hosp Jt Dis 2011;69(01):11-16

41 Noble A. Brachial plexus injuries and shoulder dystocia: medicolegal commentary and implications. J Obstet Gynaecol 2005;25 (02):105-107

42 Hickson GB, Clayton EW, Githens PB, Sloan FA. Factors that prompted families to file medical malpractice claims following perinatal injuries. JAMA 1992;267(10):1359-1363 